\title{
An inventory model for deteriorating items with seasonal products and an option of an alternative market
}

\author{
Shilpy Tayal ${ }^{\mathrm{a}^{*}}$, S.R.Singh ${ }^{\mathrm{b}}$ and Rajendra Sharma ${ }^{\mathrm{a}}$
}

${ }^{a}$ Department of mathematics, Graphic Era University, Dehradun, India

${ }^{b}$ Department of mathematics, DNPG College, Meerut, India

\section{H R O N I C L E}

\section{Article history:}

Received March 10, 2014

Received in revised format 25 July 2014

Accepted August 202014

Available online

August 222014

Keywords:

Seasonal pattern demand

Alternate market

Deterioration

Expiration date

Uncertain lead time

Partial backlogging

\begin{abstract}
A B S T R A C T
For today's high competitive market, when immensity purchasing of inventory becomes convenient or obligatory, to discover an alternative market in order to maximize the revenue earned is a tradition. In this paper, we considered an inventory model for seasonal products with Weibull rate of deterioration having two potential markets, say, primary and alternate. To handle the inventory up to the next season will result an increase in total cost. So it is a favorable task to transfer the remaining stock to the alternate market even at a slightly differ in selling price. Solution procedure, numerical examples and sensitivity analysis for different variables are presented to illustrate the model.
\end{abstract}

(C) 2014 Growing Science Ltd. All rights reserved.

\section{Introduction}

In real world situations, it is observed that many products in life have a seasonal demand pattern. So the models with seasonal demand are prevalent because of its extensive application in the inventory management of the products with short life cycles. As a type of demand, seasonal demand is extremely common but is difficult to manage, efficiently. Bradely and Arntzen (1999) developed a model for seasonal demand environment, in which they simultaneously take capacity, inventory and scheduling decisions for maximizing the return on assets. Single opportunity for procurement and single option for switching the market have been considered by Petruzzi and Monahan (2003). They consider two non-overlapping markets with selling price lower in the secondary market. You (2005) considered pricing for an inventory model with deterministic price dependent seasonal demand declining with time. Hsu et al. (2007) presented an optimal ordering decision for deteriorating items with expiration date and uncertain lead time for seasonal products. In this they discussed the items whose demand decreases as they are nearer to the expiration date. Tayal et al. (2014) presented an inventory model of seasonal products for deteriorating items in which they apply a preservation

* Corresponding author

E-mail address: agarwal_shilpy83@yahoo.com (S. Tayal)

(C) 2014 Growing Science Ltd. All rights reserved.

doi: $10.5267 /$ j.uscm.2014.8.003 
technology cost to reduce the product's rate of deterioration. In this model the occurring shortages are partially backlogged. Saha S. et al. (2010) investigated an optimal pricing and production lot-sizing policy for seasonal products over a finite horizon.

For today's high competitive market, immensity purchasing of inventory becomes convenient or obligatory and also results in significant cost reduction. Sometimes the overall circumstances are such that the supplier is influenced into buying more than he can sell in a season. Under such circumstances, he is compelled to rent another warehouse to stock the excess items or to transport them any other place where these are required. The seasons for the products may be different in different cities. For example crackers are used on different occasions at different regions in India. Woollens are start to use around September in the northern regions and then moves southwards till January. Hence, demand for different products experiences a boom at one place and slump at another place at the same time. Banerjee and Sharma (2010) investigated an inventory model for seasonal demand in which they introduced the option of an alternate market. According to the demand of the product distributor may sell the product in secondary market at higher or same price and thus the cost of warehouse and deterioration can be reduced. In general, almost all items deteriorate over time. There are many products in the real world that are subject to a significant rate of deterioration. Singh and Singh (2007) proposed an EOQ inventory model in which they considered the Weibull distribution deterioration, ramp type demand and partial backlogging. They optimized the order quantity, ordering cost, reorder point and lead time. Shukla et al. (2013) presented an EOQ model for deteriorating items with exponential demand rate and shortages. In this proposed model, shortages are allowed and partially backlogged. Tripathi and Mishra (2014) introduced an inventory model with inventory-dependent demand for deteriorating items in a single warehouse system. This paper derives a deterministic inventory model with single warehouse and shortages. Pattnaik (2013) developed an inventory model for optimization in an instantaneous economic order quantity (EOQ) incorporated with promotional effort cost, variable ordering cost and units lost due to deterioration. Singh and Sharma (2014) presented an optimal trade-credit policy for perishable items deeming imperfect production in which they considered the consumption rate as stock dependent. Tayal et al. (2014) introduced a multi item inventory model for deteriorating products and allowable shortages. In this model the effect of expiration date is discussed. Singh et al. (2009) developed an inventory model for perishable items in which they considered the power demand pattern and partial backlogging of occurring shortages. In this model, they optimized the order quantity and replenishment cycle of the product. Singh and Singh (2009) developed a production inventory model with variable demand rate for deteriorating items under permissible delay in payment. In this model a single item, single cycle economic production quantity model for perishable products is proposed where the demand is twocomponent and stock dependent. Singhal and Singh (2013) worked on a volume flexible multi items inventory system with imprecise environment for deteriorating product. The decrease or loss of utility due to decay is usually a function of the on-hand inventory. It is reasonable to note that a product may be understood to have a lifetime which ends when utility reaches zero. Price is also an important factor that influences demand. Whitin (1955) first incorporated economic price theory in inventory literature. Saha and Basu (2010) developed an inventory model for deteriorating items with ramptype time and price dependent consumption rate for seasonal product over a finite planning horizon. Singh et al. (2011) worked on a soft computing based inventory model for deteriorating items in which they considered the consumption rate as price dependent. In this study they considered the two warehouse and solve this model under inflationary environment.

In the present model, for seasonal products we consider two potential markets say primary market and alternate market. During a replenishment cycle, there may be multiple demand seasons in each market. We assume that at the end of the $\mathrm{i}^{\text {th }}$ demand season at the primary market, there is a fixed time lag, in which inventory is transported to alternate or secondary market with or without change in selling price. This option can be exercised at the end of any complete season at the primary market. Hence, there may be instances when one market experiences inter-season gap in demand while the 
other market has positive demand. After completion of each season in the primary market, the distributor has two options. Either he goes to the alternate market or stays back in the primary market. In one replenishment cycle, the distributor has a single option to exit the primary market by transferring the inventory. An integrated inventory model for vendor and buyer is presented in this paper, which is based on the integrated expected total relevant costs of both buyer and vendor. This model is beneficial for today's high competitive market. To handle the inventory up to the next season will result an increase in total cost due to the cost of deterioration and warehouses. So it is beneficial for the distributor to shift the remaining stock to the alternate market even at a slightly differ in selling price. The whole combination is very unique and very much practical. The setup has been explored numerically as well, an optimal solution has been reached at, and the sensitivity of that solution has also been checked with respect to various system parameters.

\section{Assumptions}

1. The retailers selling price per unit $\mathrm{p}$ and backorder price $p_{\mathrm{s}}$ are predetermined such that $p_{s}=$ $\lambda p>c$ where $\lambda>1$

2. Seasonal pattern demand for the product follows a deterministic function of price and season such that

$$
\begin{aligned}
d_{j}(t, p) & =\frac{a w(j)}{p_{i}^{b}}, \mathrm{j}=1,2, \ldots \ldots \ldots . . . \mathrm{N}, \\
& =0 \quad \mathrm{j}>\mathrm{N}
\end{aligned}
$$

where $\mathrm{w}(\mathrm{j})=\frac{N-j+1}{N} \quad a, b>0$

This means that the customer's demand is smaller when it is nearer to the product expiration date.

3. Shortages are allowed and if possible, partially backlogged.

4. The fraction of customers backordered is assumed to be linearly decreasing with waiting time $\eta$ and is assumed to be:-

$$
\theta(\eta)=1-\frac{\eta}{T} \quad 0 \leq \eta \leq T
$$

5. The warehouse has unlimited capacity.

6. Lead time is considered.

7. Deterioration rate is taken as a Weibull function of time.

8. At the end of each season in the primary market, the distributor has an option of transferring the remaining inventory to the alternate market and/or change the price. Once the inventory is in the alternate market, it cannot be brought back to the primary market.

9. Supplier's delivery does not go beyond the second season

10.The time lag and deterioration during transportation is negligible.

\section{Notations}

$\mathrm{p}_{1} \quad$ retailer's selling price per unit in primary market, $\mathrm{p}_{2} \quad$ retailer's selling price per unit in alternate market,

$\theta(\eta) \quad$ the fraction of customer's backordered with the condition that they receive the order after $\eta$ unit of time, 
length of seasonal interval,

$\mathrm{Q}_{0}$ retailer's order quantity for each replenishment,

$\mathrm{Q}_{1}$ retailer's sales amount without backordering over the replenishment cycle, retailer's backorder quantity at the end of the replenishment cycle, a constant inventory level during transportation,

$\mathrm{N}$ initial inventory level in primary market,

NT expiration date of product,

a critical time at which inventory level reaches zero in the last season,

$\xi$
$c$

$\mathrm{c}_{\mathrm{p}} \quad$ supplier's production cost per unit, $\mathrm{c}_{\mathrm{p}}<\mathrm{c}$

$c_{0} \quad$ retailer's ordering cost per replenishment cycle,

$\mathrm{c}_{\mathrm{h}} \quad$ unit inventory holding cost per unit time,

s unit shortage cost per unit time,

$t_{p} \quad$ cost of transportation,

$\mathrm{r}$

$\mu$

retailer's penalty cost per unit of a lost sale including loss of profit,

processing cost including making an inventory and deteriorated item per season,

y supplier's lead time,

$\mathrm{I}_{\mathrm{jP}}(\mathrm{t}) \quad$ retailer's inventory level at $\mathrm{t}$ in the primary market during the $\mathrm{j}^{\text {th }}$ season,

$\mathrm{I}_{\mathrm{jA}}(\mathrm{t})$ retailer's inventory level at $t$ in the alternate market during the $\mathrm{j}^{\text {th }}$ season,

$\mathrm{F}_{\mathrm{R}}(\mathrm{m}, \mathrm{n}, \mathrm{v}) \quad$ retailer's unit time profit,

$\mathrm{F}_{\mathrm{Rd}}(\mathrm{m}, \mathrm{n}, \mathrm{v}, \mathrm{y})$ retailer's unit time profit when $\mathrm{y}>0$.

\section{Mathematical model}

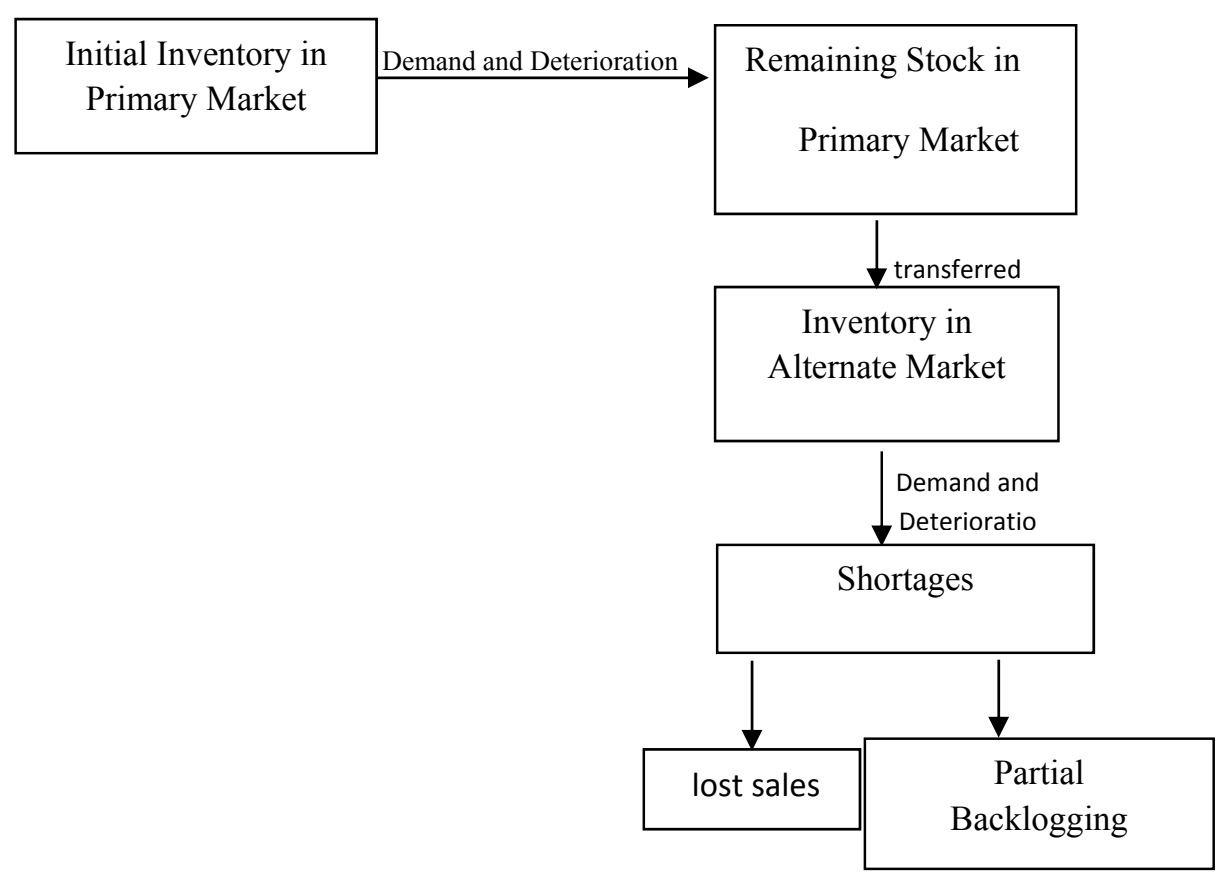

\subsection{Case 1: When $\mathrm{y} \leq 0$}

Fig. 1. Flow chart of inventory

In this case, the supplier accomplishes the order earlier than needed by the buyer. Here we assume that after completion of the season in primary market, the retailer goes to the alternate market with 
different selling price. In this case he has to bear some transportation cost. The deterioration during transportation is negligible. The inventory system of the retailer during a given cycle is showed in fig.2. The differential equation governing the transition of the system is given as follows:

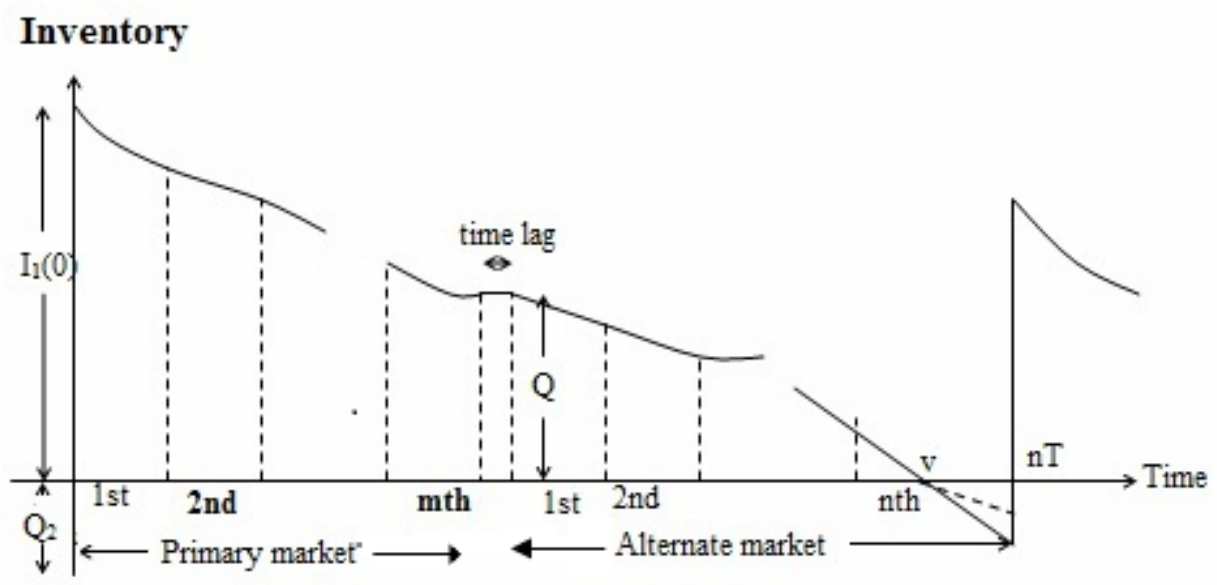

Fig. 2. Retailer's inventory system

\section{Primary market}

Inventory level during $1^{\text {st }}$ season is as follows,

$\frac{d I_{1 P}(t)}{d t}=-\alpha \beta t^{\beta-1} I_{1 P}(t)-\frac{a w(1)}{p_{1}^{b}} . \quad 0 \leq t \leq T$

Using boundary condition $I_{1 P}(0)=S$, the solution of this equation is given as follows,

$I_{1 P}(t)=\left[S-\frac{a}{p_{1}^{b}}\left(t+\frac{\alpha t^{\beta+1}}{\beta+1}\right)\right] e^{-\alpha t^{\beta}} . \quad 0 \leq t \leq T$

Inventory level during $2^{\text {nd }}$ season is as follows,

$\frac{d I_{2 P}(t)}{d t}=-\alpha \beta t^{\beta-1} I_{2 P}(t)-\frac{a w(1)}{p_{1}^{b}} . \quad 0 \leq t \leq T$

Using boundary condition $\mathrm{I}_{2 \mathrm{P}}(0)=\mathrm{I}_{1 \mathrm{P}}(\mathrm{T})$, the solution of this equation is given as follows,

$I_{2 P}(t)=-\frac{a w(2)}{p_{1}^{b}}\left(t+\frac{\alpha t^{\beta+1}}{(\beta+1)}\right) e^{-\alpha t^{\beta}}+\left\{-\frac{a w(1)}{p_{1}^{b}}\left(T+\frac{\alpha T^{\beta+1}}{\beta+1}\right)+S\right\} e^{-\alpha\left(T^{\beta}+t^{\beta}\right)}$.

Similarly the inventory level in the primary market during $\mathrm{j}^{\text {th }}$ season will be:-

$$
\begin{aligned}
& I_{j P}(t)=- \frac{a w(j)}{p_{1}^{b}}\left(t+\frac{\alpha t^{\beta+1}}{\beta+1}\right) e^{-\alpha t^{\beta}}+\sum_{l=2}^{j-1}-\frac{a w(l)}{p_{1}^{b}}\left(T+\frac{\alpha T^{\beta+1}}{\beta+1}\right) e^{-\alpha\left((j-l) T^{\beta}+t^{\beta}\right.} \\
&+\left\{-\frac{a w(1)}{p_{1}^{b}}\left(T+\frac{\alpha T^{\beta+1}}{\beta+1}\right)+S\right\} e^{-\alpha\left((j-1) T^{\beta}+t^{\beta}\right)} \quad 0 \leq t \leq T
\end{aligned}
$$

Inventory level in the primary market during the last $\left(\mathrm{m}^{\text {th }}\right)$ season is as follows,

$\frac{d I_{m P}(t)}{d t}=-\alpha \beta t^{\beta-1} I_{1 m P}(t)-\frac{a w(m)}{p_{1}^{b}} . \quad 0 \leq t \leq T$

Using boundary condition $\mathrm{I}_{\mathrm{mP}}(\mathrm{T})=\mathrm{Q}$, where $\mathrm{Q}<\mathrm{S}$, the solution of this equation is given as follows,

$$
I_{m P}(t)=\left[Q e^{\alpha T^{\beta}}+\frac{a w(m)}{p_{1}^{b}}\left\{(T-t)+\frac{\alpha}{\beta+1}\left(T^{\beta+1}-t^{\beta+1}\right)\right\}\right] e^{-\alpha t^{\beta}}, \quad 0 \leq t \leq T
$$

Now due to the completion of the season in the primary market the remaining stock is transferred to the alternate market with an additional cost of transportation. It is assumed that deterioration during transportation is negligible. 
In alternate market it will be the starting of season for this remaining stock. So the inventory level during the first season in this market is governed by the differential equation

$\frac{d I_{1 A}(t)}{d t}=-\alpha \beta t^{\beta-1} I_{1 A}(t)-\frac{a w(1)}{p_{2}^{b}} . \quad 0 \leq t \leq T$

Using boundary condition $\mathrm{I}_{1 \mathrm{~A}}(0)=\mathrm{Q}$, the solution of this equation is given as follows,

$I_{1 A}(t)=\left[Q-\frac{a}{p_{2}^{b}}\left(t+\frac{\alpha t^{\beta+1}}{\beta+1}\right)\right] e^{-\alpha t^{\beta}} . \quad 0 \leq t \leq T$

Inventory level during $2^{\text {nd }}$ season in the alternate market is given as follows,

$\frac{d I_{2 A}(t)}{d t}=-\alpha \beta t^{\beta-1} I_{2 A}(t)-\frac{a w(1)}{p_{2}^{b}} . \quad 0 \leq t \leq T$

Using boundary condition $\mathrm{I}_{2 \mathrm{~A}}(0)=\mathrm{I}_{1 \mathrm{~A}}(\mathrm{~T})$, the solution of this equation is given by the following,

$I_{2 A}(t)=-\frac{a w(2)}{p_{2}^{b}}\left(t+\frac{\alpha t^{\beta+1}}{(\beta+1)}\right) e^{-\alpha t^{\beta}}+\left\{Q-\frac{a w(1)}{p_{2}^{b}}\left(T+\frac{\alpha T^{\beta+1}}{\beta+1}\right)\right\} e^{-\alpha\left(T^{\beta}+t^{\beta}\right)} .0 \leq t \leq T$

Similarly, the inventory level in the primary market during $\mathrm{j}^{\text {th }}$ season will be

$$
\begin{array}{rlr}
I_{j A}(t)= & -\frac{a w(j)}{p_{2}{ }^{b}}\left(t+\frac{\alpha t^{\beta+1}}{\beta+1}\right) e^{-\alpha t^{\beta}}+\sum_{l=2}^{j-1}-\frac{a w(l)}{p_{2}^{b}}\left(T+\frac{\alpha T^{\beta+1}}{\beta+1}\right) e^{-\alpha\left((j-l) T^{\beta}+t^{\beta}\right.} \\
& +\left\{-\frac{a w(1)}{p_{2}{ }^{b}}\left(T+\frac{\alpha T^{\beta+1}}{\beta+1}\right)+Q\right\} e^{-\alpha\left((j-1) T^{\beta}+t^{\beta}\right)} & 0 \leq t \leq T
\end{array}
$$

Inventory level in the alternate market during the $\mathrm{n}^{\text {th }}$ season is as follows,

$\frac{d I_{n A}(t)}{d t}=-\alpha \beta t^{\beta-1} I_{n A}(t)-\frac{a w(n)}{p_{2}^{b}} \quad 0 \leq t \leq v$

Using boundary condition $\mathrm{I}_{\mathrm{nA}}(\mathrm{v})=0$, the solution of this equation is given as follows,

$I_{n A}(t)=\left[\frac{a w(n)}{p_{2}{ }^{b}}\left\{(v-t)+\frac{\alpha}{\beta+1}\left(v^{\beta+1}-t^{\beta+1}\right)\right\}\right] e^{-\alpha t^{\beta}}, \quad 0 \leq t \leq v$

$\frac{d I_{n A}(t)}{d t}=-\frac{a w(1)}{p_{2}^{b}} . \quad v \leq t \leq T$

Using boundary condition $\mathrm{I}_{\mathrm{nA}}(\mathrm{v})=0$, the solution of this equation is given as follows,

$$
I_{n A}(t)=\frac{a}{p_{2}{ }^{b}}(v-t) \quad v \leq t \leq T
$$

The replenishment cycle and shortage length are set at $n T$ and $(\mathrm{T}-\mathrm{v})$ units of time respectively.

Here two subcases arise:

a) We can consider shortages to be lost sales, that is $\eta=\mathrm{T}$, since the backlogs will be cleared when the next replenishment arrives. Replenishment arrives in the primary market. If backlog occurs in the alternate market then logistic restriction do not allow it.

b) If backlogs are cleared then the backorder price will include the transportation cost. In this case $\lambda p_{2}>p_{2} \Rightarrow \lambda>1$

\section{Subcase (a): When shortages without backordering occur:}

In this case shortage without backordering will occur so all the shortages will be considered as lost sale. So the retailer's unit time profit without late delivery is:- 
$\mathrm{F}_{\mathrm{R}}(\mathrm{m}, \mathrm{n}, \mathrm{v})=\frac{1}{(m+n) T}[$ sales revenue-purchasing cost-holding cost- deterioration cost-lost sale cost -transportation cost-ordering cost]

$$
=\frac{1}{(m+n) T}\left[\mathrm{R}(\mathrm{m}, \mathrm{n}, \mathrm{v})-\mathrm{C}(\mathrm{m}, \mathrm{n}, \mathrm{v})-\mathrm{H}(\mathrm{m}, \mathrm{n}, \mathrm{v})-\mathrm{D}(\mathrm{m}, \mathrm{n}, \mathrm{v})-\mathrm{L}(\mathrm{m}, \mathrm{n}, \mathrm{v})-\mathrm{t}_{\mathrm{p}}-\mathrm{c}_{\mathrm{o}}\right]
$$

Sales revenue:

$\mathrm{R}(\mathrm{m}, \mathrm{n}, \mathrm{v})=\mathrm{p}_{1} \mathrm{Q}_{\mathrm{P}}+\mathrm{p}_{2} \mathrm{Q}_{\mathrm{A}}$

$\mathrm{p}_{1} \mathrm{Q}_{\mathrm{P}}=\sum_{j=1}^{m} p_{1} \int_{0}^{T} d_{j}(t, p) . d t$

$\mathrm{p}_{1} \mathrm{Q}_{\mathrm{P}}=\frac{a m p_{1}}{N p_{1}^{b}}(2 N-m+1)$

$\mathrm{p}_{2} \mathrm{Q}_{\mathrm{A}}=\sum_{j=1}^{n-1} p_{2} \int_{0}^{T} d_{j}(t, p) \cdot d t+p_{2} \int_{0}^{v} d_{n}(t, p) \cdot d t$

$\mathrm{p}_{2} \mathrm{Q}_{\mathrm{A}}=\frac{a T p_{2}}{2 N p_{2}^{b}}(n-1)(2 N-n+2)+\frac{a p_{2}}{N p_{2}^{b}}(N-n+1) \cdot v$

The ordering quantity at each replenishment is:

$\mathrm{Q}_{\mathrm{o}}=\mathrm{I}_{1 \mathrm{P}}(0)$

Purchasing cost:-

$\mathrm{C}(\mathrm{m}, \mathrm{n}, \mathrm{v})=\mathrm{I}_{1 \mathrm{P}}(0) . \mathrm{c}$

The lost sale amount $=\int_{v}^{T} d_{1}(t, p)(1-\theta(T)) d t$

L.S.C. $=\frac{a}{p_{2}{ }^{b}}(T-v) r$

Processing cost:

$\mathrm{B}(\mathrm{m}, \mathrm{n}, \mathrm{v})=(\mathrm{m}+\mathrm{n}) \mu$

Holding cost:

$$
\begin{aligned}
& H(m, n, v)=\sum_{j=1}^{m-1} H_{j P}(T)+H_{m P}(T)+\sum_{j=1}^{n-1} H_{j A}(T)+H_{n A}(t) \quad \text { where }(\mathrm{m} \& \mathrm{n}>1) \\
& H(m, n, v)=c_{h} \sum_{j=1}^{m-1} \int_{0}^{T} I_{j P}(t) d t+c_{h} \int_{0}^{T} I_{m P}(t) d t+c_{h} \sum_{j=1}^{n-1} \int_{0}^{T} I_{j A}(t) d t+c_{h} \int_{0}^{v} I_{n A}(t) d t \\
& H_{j P}{ }^{T}=-\frac{c_{h} \alpha w(j)}{p_{1}^{b}}\left(\frac{T^{2}}{2}+\frac{\alpha T^{\beta+2}}{(\beta+1)(\beta+2)}-\frac{\alpha T^{\beta+2}}{(\beta+2)}\right)+c_{h} \sum_{l=2}^{j-1}-\frac{a w(l)}{p^{b}}\left(T+\frac{\alpha T^{\beta+1}}{\beta+1}\right) \\
& \quad\left\{T-\alpha\left((j-l) T^{\beta+1}+\frac{T^{\beta+1}}{\beta+1}\right)\right\}+c_{h}\left\{-\frac{a}{p^{b}}\left(T+\frac{\alpha T^{\beta+1}}{\beta+1}\right)+S\right\}\left\{T-\alpha\{j-1\} T^{\beta+1}+\frac{T^{\beta+1}}{\beta+1}\right\} \\
& H_{m P}{ }^{T}=c_{h}\left\{Q e^{\alpha T^{\beta}} T+\frac{a w(m)}{p_{1}^{b}} \frac{T^{2}}{2}+\frac{\alpha}{\beta+2} T^{\beta+2}-\alpha Q e^{\alpha T^{\beta}} \frac{T^{\beta+1}}{\beta+1}-\frac{\alpha a w(m)}{p_{1}^{b}}\left(\frac{T^{\beta+2}}{(\beta+1)(\beta+2)}\right)\right\} \\
& H_{n A}{ }^{v}=c_{h} \frac{a w(n)}{p_{2}{ }^{b}}\left\{\frac{v^{2}}{2}+\frac{\alpha}{(\beta+2)} v^{\beta+2}-\frac{\alpha v^{\beta+2}}{(\beta+1)(\beta+2)}\right\} \\
& H_{j A}{ }^{T}=-\frac{c_{h} \alpha w(j)}{p_{2}^{b}}\left(\frac{T^{2}}{2}+\frac{\alpha T^{\beta+2}}{(\beta+1)(\beta+2)}-\frac{\alpha T^{\beta+2}}{(\beta+2)}\right)+c_{h} \sum_{l=2}^{j-1}-\frac{a w(l)}{p_{2}^{b}}\left(T+\frac{\alpha T^{\beta+1}}{\beta+1}\right)
\end{aligned}
$$


where

$$
\left\{T-\alpha\left((j-l) T^{\beta+1}+\frac{T^{\beta+1}}{\beta+1}\right)\right\}+c_{h}\left\{-\frac{a}{p_{2}^{b}}\left(T+\frac{\alpha T^{\beta+1}}{\beta+1}\right)+Q\right\}\left\{T-\alpha\{j-1\} T^{\beta+1}+\frac{T^{\beta+1}}{\beta+1}\right\},
$$

$$
\begin{aligned}
\mathrm{H}(1,1, \mathrm{v})= & c_{h}\left\{Q e^{\alpha T^{\beta}} T+\frac{\alpha}{p_{1}^{b}} \frac{T^{2}}{2}+\frac{\alpha}{\beta+2} T^{\beta+2}-\alpha Q e^{\alpha T^{\beta}} \frac{T^{\beta+1}}{\beta+1}-\frac{\alpha a}{p_{1}^{b}} \frac{T^{\beta+2}}{(\beta+1)(\beta+2)}\right\} \\
& +c_{h} \frac{a}{p_{2}^{b}}\left(\frac{v^{2}}{2}+\frac{\alpha}{\beta+2} v^{\beta+2}-\frac{\alpha v^{\beta+2}}{(\beta+1)(\beta+2)}\right) .
\end{aligned}
$$

Deteriorated items=Total initial stock - Total demand(during positive inventory)

Det.cost $\left.=I_{1 P}(0)-\frac{a}{N p_{1}{ }^{b}} m(2 N-m+1)-\frac{a T}{2 N p_{2}{ }^{b}}(n-1)(2 N-n+2)-\frac{a}{N p_{2}{ }^{b}}(N-n+1) v\right\} d$

Transportation cost $=\mathrm{t}_{\mathrm{p}}$

Ordering $\cos \mathrm{t}=\mathrm{c}_{\mathrm{o}}$

Subcase(b): When backlogs are cleared:

The retailer's unit time profit without late delivery when shortages occur and partially backlogged:$\mathrm{F}_{\mathrm{R}}(\mathrm{m}, \mathrm{n}, \mathrm{v})=\frac{1}{(m+n) T}[$ sales revenue-purchasing cost-holding cost- deterioration cost-lost sale

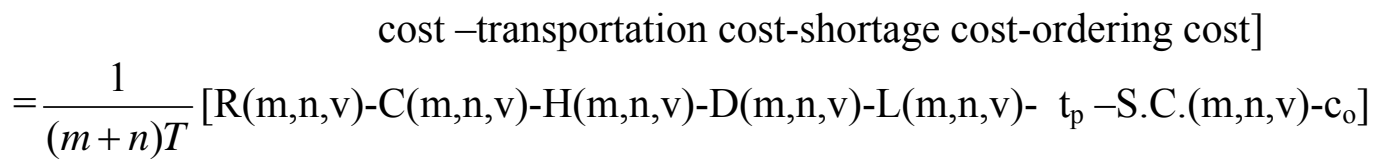

Sales revenue:

$\mathrm{R}(\mathrm{m}, \mathrm{n}, \mathrm{v})=\mathrm{p}_{1} \mathrm{Q}_{\mathrm{P}}+\mathrm{p}_{2} \mathrm{Q}_{\mathrm{A}}+\lambda \mathrm{p}_{2} \mathrm{Q}_{2 \mathrm{~A}}$

where:-

$\mathrm{p}_{1} \mathrm{Q}_{\mathrm{P}}=\sum_{j=1}^{m} p_{1} \int_{0}^{T} d_{j}(t, p) \cdot d t$

$\mathrm{p}_{2} \mathrm{Q}_{\mathrm{A}}=\sum_{j=1}^{n-1} p_{2} \int_{0}^{T} d_{j}(t, p) \cdot d t+p_{2} \int_{0}^{v} d_{n}(t, p) \cdot d t$

$Q_{2 A}=\int_{v}^{T} d_{1}(t, p) \theta(T-t) d t$

The order quantity at each replenishment is:-

$\mathrm{Q}_{\mathrm{o}}=\left(\mathrm{I}_{1 \mathrm{P}}(0)+\mathrm{Q}_{2 \mathrm{~A}}\right)$

Purchasing cost:-

$\mathrm{C}(\mathrm{m}, \mathrm{n}, \mathrm{v})=\left(\mathrm{I}_{1 \mathrm{P}}(0)+\mathrm{Q}_{2}\right) \cdot \mathrm{c}$

The lost sale amount $=\int_{v}^{T} d_{1}(t, p)(1-\theta(T-t)) d t$

L.S.C. $=\frac{a}{p_{2}{ }^{b}}\left(\frac{T}{2}+\frac{v^{2}}{2 T}-v\right) r$

Processing cost:

$\mathrm{B}(\mathrm{m}, \mathrm{n}, \mathrm{v})=(\mathrm{m}+\mathrm{n}) \mu$ 
Holding cost:

Holding cost will be the same as in subcase (a).

$H(m, n, v)=\sum_{j=1}^{m-1} H_{j P}(T)+H_{m P}(T)+\sum_{j=1}^{n-1} H_{j A}(T)+H_{n A}(t) \quad$ where $(\mathrm{m} \& \mathrm{n}>1)$

Deteriorated items=Total initial stock - Total demand(during positive inventory)

Det.cost $\left.=I_{1 P}(0)-\frac{a}{N p_{1}^{b}} m(2 N-m+1)-\frac{a T}{2 N p_{2}^{b}}(n-1)(2 N-n+2)-\frac{a}{N p_{2}^{b}}(N-n+1) v\right\} d$.

Transportation cost $=t_{p}$

Shortage cost $=s \int_{v}^{T}-I_{n A}(t) d t$

$$
=s \frac{a}{p_{2}^{b}}\left(\frac{T^{2}}{2}+\frac{v^{2}}{2}-v T\right)
$$

Ordering cost $=\mathrm{c}_{\mathrm{o}}$

Case 4.2: When the supplier's lead time $y>0$ :

Subcase(a): When shortages without backlogging occurs:

$$
\begin{array}{r}
\mathrm{F}_{\mathrm{Rd}}(\mathrm{m}, \mathrm{n}, \mathrm{v}, \mathrm{y})=\frac{1}{(m+n) T}[\mathrm{Rd}(\mathrm{m}, \mathrm{n}, \mathrm{v}, \mathrm{y})-\mathrm{Cd}(\mathrm{m}, \mathrm{n}, \mathrm{v}, \mathrm{y})-\mathrm{Ld}(\mathrm{m}, \mathrm{n}, \mathrm{v}, \mathrm{y})- \\
\left.(\mathrm{B}, \mathrm{m}, \mathrm{n}, \mathrm{v})-\mathrm{Hd}-\mathrm{C}_{\mathrm{o}}-\text { D.C.(m,n,v,y) }-\mathrm{t}_{\mathrm{p}}\right]
\end{array}
$$

We assume that the shortages without backordering will occur during the interval[0,y], since the lead time is uncertain.

$\mathrm{Q}_{1 \mathrm{~d}}=\sum_{j=1}^{m} \int_{0}^{T} d_{j}(t, p) \cdot d t+\sum_{j=1}^{n-1} \int_{0}^{T} d_{j}(t, p) \cdot d t+\int_{0}^{v} d_{n}(t, p) \cdot d t-\int_{0}^{y} d_{1}(t, p) d t$

$\mathrm{Q}_{1 \mathrm{~d}}=\frac{a m}{N p_{1} b}(2 N-m+1)+\frac{a T}{2 N p_{2} b}(n-1)(2 N-n+2)+\frac{a}{N p_{2} b}(N-n+1) \cdot v-\frac{a y}{p_{1}^{b}}$,

Purchasing cost:-

$\mathrm{Cd}(\mathrm{m}, \mathrm{n}, \mathrm{v}, \mathrm{y})=\mathrm{I}_{1 \mathrm{P}}(\mathrm{y}) . \mathrm{c}$

Since in this case during stock out the complete demand will be the lost sale. So the lost sale amount will be:

Lost sale amount $=\int_{0}^{y} d_{1}(t, p) d t+\int_{v}^{T} d_{1}(t, p) d t$.

$\operatorname{Ld}(\mathrm{m}, \mathrm{n}, \mathrm{v}, \mathrm{y})=\frac{a}{p_{1}^{b}} y r+\frac{a}{p_{2}^{b}}(T-v) r$,

Processing cost:

$\mathrm{B}(\mathrm{m}, \mathrm{n}, \mathrm{v})=(\mathrm{m}+\mathrm{n}) \mu$

Holding cost:

$\operatorname{Hd}(\mathrm{n}, \mathrm{m}, \mathrm{v}, \mathrm{y})=\sum_{j=1}^{m-1} H_{j P}(T)+H_{m P}(T)+\sum_{j=1}^{n-1} H_{j A}(T)+H_{n A}(v)-h \int_{0}^{y} I_{1 P}(t) d t$ 
where:

$\operatorname{Hd}(1,1, \mathrm{v}, \mathrm{y})=H_{1 P}(T)+H_{1 A}(v)-h \int_{0}^{y} I_{1 P}(t) d t$,

Det.cost $=\left\{I_{1 P}(y)-\frac{a}{N p_{1}^{b}} m(2 N-m+1)-\frac{a T}{2 N p_{2}{ }^{b}}(n-1)(2 N-n+2)-\frac{a}{N p_{2}^{b}}(N-n+1) v\right\} d$,

Transportation cost $=\mathrm{t}_{\mathrm{p}}$

Ordering cost $=\mathrm{C}_{\mathrm{o}}$

Subcase(b): when shortages are partially backlogged:

The retailer's unit time profit with late delivery by $y$ unit time $F_{R d}(m, n, v, y)$ is:-

$$
\begin{gathered}
\mathrm{F}_{\mathrm{Rd}}(\mathrm{m}, \mathrm{n}, \mathrm{v}, \mathrm{y})=\frac{1}{(m+n) T}[\mathrm{Rd}(\mathrm{m}, \mathrm{n}, \mathrm{v}, \mathrm{y})-\mathrm{Cd}(\mathrm{m}, \mathrm{n}, \mathrm{v}, \mathrm{y})-\mathrm{Ld}(\mathrm{m}, \mathrm{n}, \mathrm{v}, \mathrm{y})-(\mathrm{B}, \mathrm{m}, \mathrm{n}, \mathrm{v})- \\
\left.\mathrm{Hd}-\mathrm{C}_{\mathrm{o}}-\text { D.C.(m,n,v,y) - S.C.(m,n,v,y) }-\mathrm{t}_{\mathrm{p}}\right] .
\end{gathered}
$$

We assume that the shortages without backordering will occur during the interval[0,y], since the lead time is uncertain.

$$
\begin{aligned}
\mathrm{Q}_{1 \mathrm{~d}} & =\sum_{j=1}^{m} \int_{0}^{T} d_{j}(t, p) \cdot d t+\sum_{j=1}^{n-1} \int_{0}^{T} d_{j}(t, p) \cdot d t+\int_{0}^{v} d_{n}(t, p) \cdot d t-\int_{0}^{y} d_{1}(t, p) d t \\
\mathrm{Q}_{1 \mathrm{~d}} & =\frac{a m}{N p_{1} b}(2 N-m+1)+\frac{a T}{2 N p_{2} b}(n-1)(2 N-n+2)+\frac{a}{N p_{2} b}(N-n+1) \cdot v-\frac{a y}{p_{1}^{b}} \\
Q_{2} & =\frac{a}{p_{2}{ }^{b} T}\left(\frac{T^{2}}{2}-\frac{v^{2}}{2}\right)
\end{aligned}
$$

Sales Revenue:-

$$
\begin{aligned}
\operatorname{Rd}(\mathrm{m}, \mathrm{n}, \mathrm{v}, \mathrm{y})= & \frac{p_{1} a m}{N p_{1} b}(2 N-m+1)+\frac{p_{2} a T}{2 N p_{2} b}(n-1)(2 N-n+2)+\frac{p_{2} a}{N p_{2} b}(N-n+1) \cdot v \\
& -\frac{p_{1} a y}{p_{1}^{b}}+\frac{\lambda p_{2} a}{p_{2}{ }^{b} T}\left(\frac{T^{2}}{2}-\frac{v^{2}}{2}\right)
\end{aligned}
$$

Purchasing cost:

$\mathrm{Cd}(\mathrm{m}, \mathrm{n}, \mathrm{v}, \mathrm{y})=\left(\mathrm{I}_{1 \mathrm{P}}(\mathrm{y})+\mathrm{Q}_{2}\right) \cdot \mathrm{c}$

Lost sale amount $=\int_{0}^{y} d_{1}(t, p) d t+\int_{v}^{T} d_{1}(t, p)(1-\theta(T-t)) d t$

$L d(m, n, v, y)=\frac{a}{p_{1}^{b}} y r+\frac{a}{p_{2}^{b}}\left(\frac{T}{2}+\frac{v^{2}}{2 T}-v\right) r$

Processing cost:

$B(m, n, v)=(m+n) \mu$

Holding cost:

$\mathrm{Hd}(\mathrm{m}, \mathrm{n}, \mathrm{v}, \mathrm{y})=\sum_{j=1}^{m-1} H_{j P}(T)+H_{m P}(T)+\sum_{j=1}^{n-1} H_{j A}(T)+H_{n A}(v)-h \int_{0}^{y} I_{1 P}(t) d t$, where $m \geq 2, n \geq 2$ 
Det. cost Transportation cost and ordering cost will be the same as in previous subcase.

Shortage cost $=s \frac{a}{p_{2}^{b}}\left(v T-\frac{T^{2}}{2}-\frac{v^{2}}{2}\right)$.

Since there is a potential lose $F_{R}(m, n, v)-F_{R d}(m, n, v, y)$ on the retailer due to the suppliers delay, the supplier has to compensate the retailer. Therefore the retailer's unit time profit is:-

$F_{R d}(m, n, v, y)+\left[F_{R}(m, n, v)-F_{R d}(m, n, v, y)\right]=F_{R}(m, n, v)$

ie the retailer maintains his profit regardless of the supplier's delivery behaviour.

If the retailer determines the order quantity independently, then the problem can be formulated as follows:

$\max : F_{R}(m, n, v)$

subject to: $m+n \leq N, 0<v \leq T$

Here the retailer's unit time profit is the function of variables $m, n$ and $v$, where $m$ and $n$ are discrete variables and $\mathrm{v}$ is a real number.

Since $F_{R}(m, n, v)$ is a concave function of $v$ for fixed $m$ and $n$ (Hsu et al., 2006), we can derive the optimal $v$ by solving the equation $\frac{\partial F_{R}(m, n, v)}{\partial v}=0$. Let $v(m, n)$ be the solution and $v^{*}(m, n)=$ $\min \{v(m, n), T\}$. Since the integer variables $m$ and $n$ cannot be founded by analytic method, the following search procedure is used.

\section{Solution search procedure}

Step1: Set $n=1, m=1$

Step2: If $m+n \leq N$, go to step 3-5.

Step3: Solve the equation $\frac{\partial F_{R}(m, n, v)}{\partial v}=0$ to obtain $v(n)$ and $v^{*}(n)$

Step4: Calculate $F_{R}\left(m, n, v^{*}(n)\right)$ (according to the lead time and backlogging condition)

Step5: If $F_{R}\left(m, n, v^{*}(m, n)\right)>F_{R}\left(m, n, v^{*}\right)$, let $F^{*}{ }_{R}=F_{R}\left(m, n, v^{*}(m, n)\right), m^{*}=m, n *=n$ and $v^{*}=v$

\subsection{Numerical illustration}

The above model can be illustrated by the given numerical example where the system parameters are observed as follows:

$\mathrm{N}=10, \mathrm{a}=3000, \mathrm{~b}=1.2, \mathrm{~S}=5000$ unit, $\mathrm{T}=50, \mathrm{r}=16 /$ unit, $c_{h}=0.005 /$ unit/unit time, $\mathrm{d}=30 / \mathrm{unit}, \mathrm{t}_{\mathrm{p}}=500$, $\mathrm{s}=12 /$ unit, $\mathrm{c}_{\mathrm{o}}=500 /$ order, $\mathrm{p}_{1}=45 /$ unit, $\mathrm{p}_{2}=47 /$ unit, $\lambda=1.2, \mu=20 /$ season, $\mathrm{c}=30 /$ unit $, \alpha=.0001, \beta=1.5$

With these values we find the different solutions of the system. The optimal solution exist for $\mathbf{m}=\mathbf{2}$ and $n=5$. The optimal value of unit time profit is $51989.9(y \leq 0)$ and $51963.2(y>0)$ and optimal value of $Q$ and $v$ are $\mathbf{1 8 0 8 . 5 5}$ units and $\mathbf{4 8 . 2 3 7 5}$ days respectively.

With a given value of $\mathrm{m}$ and $\mathrm{n}$, the domain $\{\{v\}: 0<v \leq T\}$ leads to the existence of the optimal $\mathrm{F}$. This occurs either at the relative extreme point or at the boundary of the domain .Hence, if the critical point $v^{*}$ belong to the compact domain, then $\mathrm{F}$ is maximum at $v=v^{*}$, if critical point $v^{*}$ does not belong to the domain, then $F$ will have maximum at the boundary. However, we need only to investigate the boundary due to the nature of problem that maximizes the function $F(v)=F(m, n, v) \quad 0<\mathrm{v} \leq \mathrm{T}$.

From Theorem 1, the optimal $v^{*}$ can be derived by setting $\frac{\partial F(v)}{\partial v}=0$, for concave $F(v)$.

5.2. Theorem1: $F(v)$ is concave in $v$. 
Proof: For given values of $m \& n$, proof is given in appendix A.

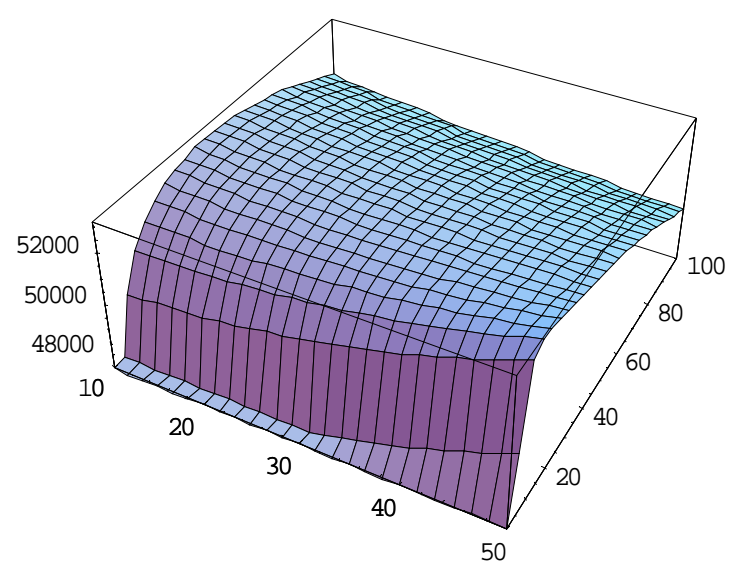

Fig. 3. concavity of the profit function with respect to ' $v$ '

Subcase(a): When backlogs are not cleared

\section{Table 1}

Solution search results for retailer

\begin{tabular}{|c|c|c|c|c|c|}
\hline $\mathrm{m}$ & $\mathrm{n}$ & $Q$ & $\mathrm{~V}$ & $\mathrm{~F}_{\mathrm{R}}($ case- 1$)$ & $\mathrm{F}_{\mathrm{Rd}}$ (case-2) \\
\hline \multirow{9}{*}{1} & 1 & 3300.21 & 42.2676 & - & - \\
\hline & 2 & 3300.21 & 42.9078 & 31455.7 & 31391 \\
\hline & 3 & 3300.21 & 43.5478 & 47586.7 & 47538.4 \\
\hline & 4 & 3300.21 & 94.1879 & 56775.3 & 56736.7 \\
\hline & 5 & 3300.21 & 44.8279 & 62461.1 & 62428.9 \\
\hline & 6 & 3300.21 & 45.4678 & 66118.8 & 66091.2 \\
\hline & 7 & 3300.21 & 46.1077 & 68486.6 & 68462.4 \\
\hline & 8 & 3300.21 & 46.7475 & 69975 & 69953.4 \\
\hline & 9 & 3300.21 & 47.3873 & 70831.2 & 70811.4 \\
\hline \multirow{8}{*}{2} & 1 & 1808.55 & 42.2676 & 49899.1 & 49837.7 \\
\hline & 2 & 1808.55 & 42.9078 & 51117.9 & 51072.1 \\
\hline & 3 & 1808.55 & 43.5478 & 51710 & 51673.5 \\
\hline & 4 & 1808.55 & 44.1879 & 51956.2 & 51925.8 \\
\hline & 5 & 1808.55 & 44.8279 & 51977.6 & 51952.1 \\
\hline & 6 & 1808.55 & 45.4678 & 51835.4 & 51812.5 \\
\hline & 7 & 1808.55 & 46.1077 & 51564.1 & 51543.7 \\
\hline & 8 & 1808.55 & 46.7475 & 51185.1 & 51166.7 \\
\hline
\end{tabular}

In this case with the given data the optimal result exist for $\mathbf{m}^{*}=\mathbf{2}$ and $\mathbf{n} * \mathbf{5}$. The optimal net profit is obtained as $\mathbf{F}^{*}=\mathbf{\$ 5 1 9 7 7 . 6}$. The optimal value of $\mathrm{v}$ comes out to be $\mathbf{v}=\mathbf{4 4 . 8 2 7 9}$ days, and the optimal net profit when $y \geq 0$ is obtained as $\mathbf{\$ 5 1 9 5 2 . 1}$

\section{Sensitivity analysis}

Corresponding to the optimal values of the system, a sensitivity analysis is carried out with respect to various system parameters. Sensitivity analysis is carried out when the parameters $\mathrm{N}$, a, band $\alpha$ changes. Table 4-7 show the changes in $\mathrm{Q}, \mathrm{v}$ and $\mathrm{F}_{\mathrm{R}}$ for variables $\mathrm{N}, \mathrm{a}, \mathrm{b}$ and $\alpha$ respectively, when $\mathrm{m}$ and $\mathrm{n}$ are fixed. $\mathrm{N}=10, \mathrm{a}=3000, \mathrm{~b}=1.2, \mathrm{~S}=5000$ unit, $\mathrm{T}=50, \mathrm{r}=16 / \mathrm{unit}, c_{h}=0.005$ /unit/unit time, $\mathrm{d}=30 /$ unit $\mathrm{t}_{\mathrm{p}}=500, \mathrm{~s}=12 /$ unit, $\mathrm{c}_{\mathrm{o}}=500 /$ order, $\mathrm{p}_{1}=45 /$ unit, $\mathrm{p}_{2}=47 /$ unit, $\lambda=1.2, \mu=20 /$ season, $\mathrm{c}=30 /$ unit, $\alpha=.0001, \beta=1.5$ 
Table 2

Sensitivity analysis for the parameter a

\begin{tabular}{lccc}
\hline $\mathrm{a}$ & $\mathrm{Q}$ & $\mathrm{V}$ & $\mathrm{F}_{\mathrm{R}}$ \\
\hline 1500 & 3227.5 & 44.8279 & 36734.9 \\
2000 & 2754.52 & 44.8279 & 44299.5 \\
2500 & 2281.53 & 44.8279 & 49380.3 \\
3000 & 1808.55 & 44.8279 & 51977.6 \\
3500 & 1335.57 & 44.8279 & 52091.3 \\
4000 & 862.584 & 44.8279 & 49721.2 \\
4500 & 389.601 & 44.8279 & 44867.5 \\
\hline
\end{tabular}

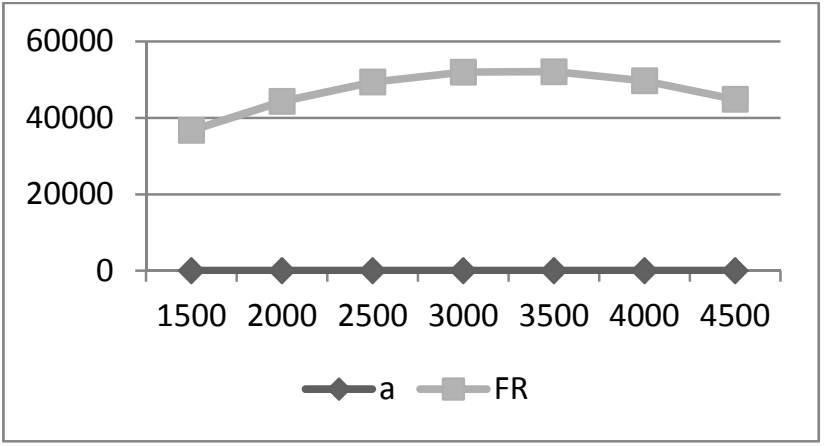

Variation in $F_{R}$ with the variation in a

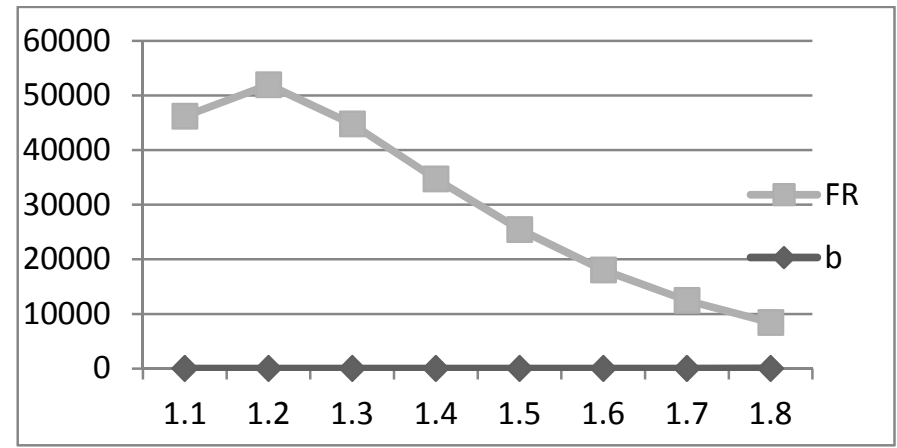

Variation in $F_{R}$ with the variation in $b$

Table 3

Sensitivity analysis for the parameter $b$

\begin{tabular}{lccc}
\hline $\mathrm{b}$ & $\mathrm{Q}$ & $\mathrm{V}$ & $\mathrm{F}_{\mathrm{R}}$ \\
\hline 1.1 & 493.868 & 44.8279 & 46215.8 \\
1.2 & 1808.55 & 44.8279 & 51977.6 \\
1.3 & 2707.01 & 44.8279 & 44788.3 \\
1.4 & 3321.02 & 44.8279 & 34723.6 \\
1.5 & 3740.65 & 44.8279 & 25465.3 \\
1.6 & 4027.42 & 44.8279 & 18041.8 \\
1.7 & 4223.4 & 44.8279 & 12465.8 \\
1.8 & 4357.33 & 44.8279 & 8426.29 \\
\hline
\end{tabular}

\section{Table 4}

Sensitivity analysis for the parameter $\alpha$

\begin{tabular}{lccc}
\hline$\alpha$ & $\mathbf{Q}$ & $\mathbf{v}$ & $\mathbf{F}_{\mathbf{R}}$ \\
\hline 0.00006 & 1901.41 & 44.8279 & 54458.2 \\
0.00008 & 1854.89 & 44.8279 & 53206 \\
0.0001 & 1808.55 & 44.8279 & 51977.6 \\
0.00012 & 1762.39 & 44.8279 & 53350.2 \\
0.00014 & 1716.41 & 44.8279 & 52533.3 \\
0.00016 & 1670.61 & 44.8279 & 51721.5 \\
0.00018 & 1624.99 & 44.8279 & 50914.8 \\
0.0002 & 1579.56 & 44.8279 & 46189.3 \\
\hline
\end{tabular}

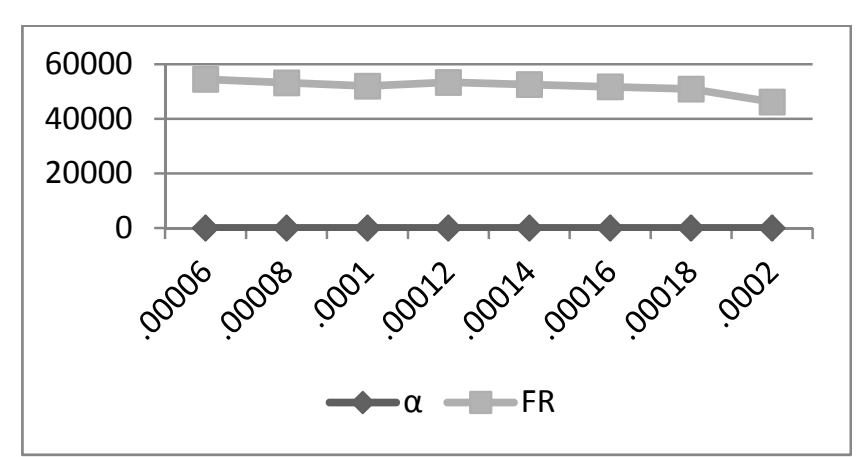

Variation in $F_{R}$ with the variation in $\alpha$

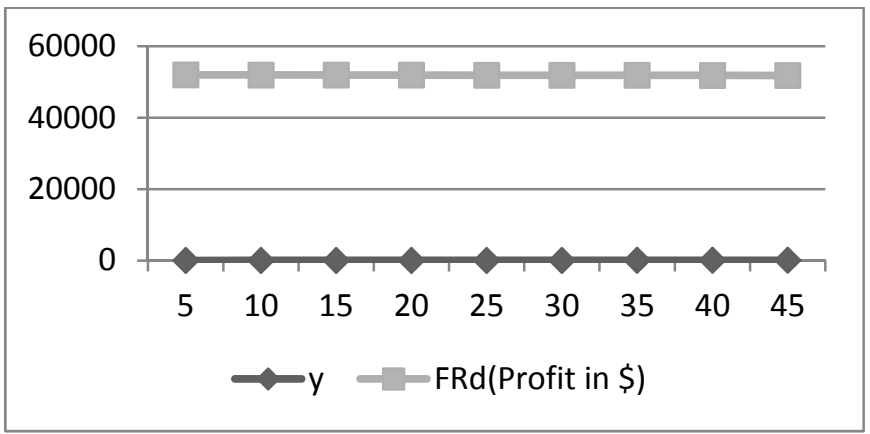

Variation in $F_{R d}$ with the variation in $y$ 
Table 5

Sensitivity analysis for the parameter $\mathrm{y}(\mathrm{y}>0)$

\begin{tabular}{lccc}
\hline $\mathrm{y}$ & $\mathrm{Q}$ & $\mathrm{v}$ & $\mathrm{F}_{\mathrm{Rd}}($ Profit in $\$)$ \\
\hline 5 & 1808.55 & 45.8279 & 51952.1 \\
10 & 1808.55 & 45.8279 & 51926.6 \\
15 & 1808.55 & 45.8279 & 51901.5 \\
20 & 1808.55 & 45.8279 & 51876.8 \\
25 & 1808.55 & 45.8279 & 51852.4 \\
30 & 1808.55 & 45.8279 & 51828.3 \\
35 & 1808.55 & 45.8279 & 51804.3 \\
40 & 1808.55 & 45.8279 & 51780.5 \\
45 & 1808.55 & 45.8279 & 51756.9 \\
\hline
\end{tabular}

Subcase(b): When backlogs are cleared.

Table 6

Solution search results for optimal values of $m \& n$ :

\begin{tabular}{cccccc}
\hline $\mathrm{m}$ & $\mathrm{n}$ & $\mathrm{Q}$ & $\mathrm{V}$ & $\mathrm{F}_{\mathrm{R}}$ & $\mathrm{F}_{\mathrm{RD}}$ \\
\hline & 1 & 3300.21 & 45.4831 & - & - \\
& 2 & 3300.21 & 46.1718 & 31493.1 & 31433.2 \\
\multirow{3}{*}{1} & 3 & 3300.21 & 46.8604 & 47611.9 & 47566.3 \\
& 4 & 3300.21 & 47.549 & 56793.4 & 56756.1 \\
& 5 & 3300.21 & 48.2375 & 62473.7 & 62442.5 \\
& 6 & 3300.21 & 48.926 & 66127.7 & 66100.6 \\
\hline & 7 & 3300.21 & 49.6143 & 68492.6 & 68468.6 \\
\hline & 1 & 1808.55 & 45.4831 & 49943.9 & 49884.8 \\
& 2 & 1808.55 & 46.1718 & 51148.7 & 51103.7 \\
& 3 & 1808.55 & 46.8604 & 51732.3 & 51695.8 \\
& 4 & 1808.55 & 47.549 & 51972.7 & 51941.9 \\
& $\mathbf{5}$ & $\mathbf{1 8 0 8 . 5 5}$ & $\mathbf{4 8 . 2 3 7 5}$ & 51989.9 & 51963.2 \\
\end{tabular}

With the given data, the optimal solution for supply chain is obtained by using software MATHEMATICA. Optimal result exist for $\mathbf{m}^{*=2}$ and $\mathbf{n}^{*}=5$. The optimal net profit of the supply chain is obtained as $\mathbf{F}^{*}=\mathbf{\$ 5 1 9 8 9}$. The optimal value of $\mathrm{v}$ comes out to be $\mathbf{v}=\mathbf{4 8 . 2 3 7 5}$ days, and the optimal net profit of the supply chain when $y \geq 0$ is obtained as $\mathbf{\$ 5 1 9 6 3 . 2}$.

\section{Table 7}

Sensitivity analysis for the parameter N

\begin{tabular}{lcccc}
\hline $\mathrm{N}$ & $\mathrm{Q}$ & $\mathrm{V}$ & \\
\hline 10 & 1808.55 & 48.2375 & \\
15 & 1757.78 & 47.3195 & 51989.9 \\
20 & 1732.4 & 46.8604 & 51323.9 \\
25 & 1717.17 & 46.585 & 50990.7 & 50790.7 \\
30 & 1702.02 & 46.4013 & 50578.6 & 5051.9 \\
35 & 1699.76 & 46.2702 & \\
\hline
\end{tabular}

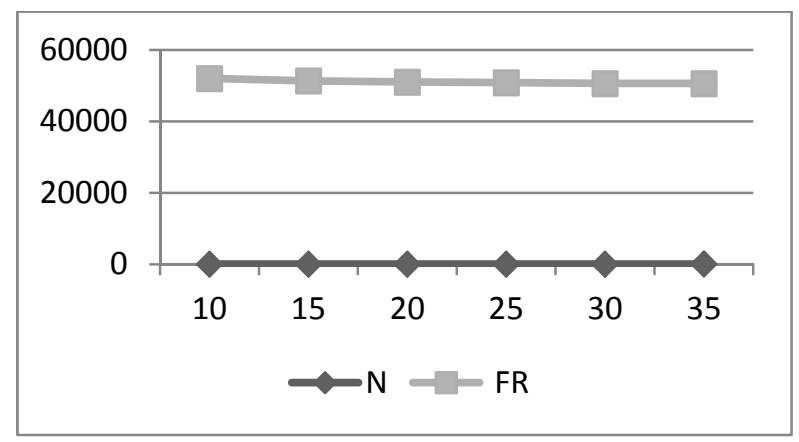

Variation in $F_{R}$ with the variation in $N$

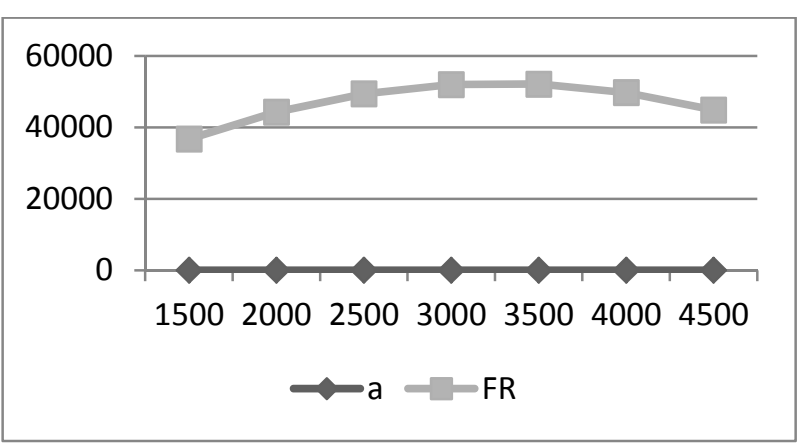

Variation in $F_{R}$ with the variation in a 
Table 8

Sensitivity analysis for the parameter a

\begin{tabular}{lccc}
\hline $\mathrm{a}$ & $\mathrm{Q}$ & $\mathrm{V}$ & $\mathrm{F}_{\mathrm{R}}$ \\
\hline 1500 & 3227.5 & 48.2375 & 36739.5 \\
2000 & 2754.52 & 48.2375 & 44306.6 \\
2500 & 2281.53 & 48.2375 & 49390 \\
3000 & 1808.55 & 48.2375 & 51989.9 \\
3500 & 1335.57 & 48.2375 & 52106.1 \\
4000 & 862.584 & 48.2375 & 49738.6 \\
4500 & 389.601 & 48.2375 & 44887.5 \\
\hline
\end{tabular}

\section{Table 9}

Sensitivity analysis for the parameter $b$

\begin{tabular}{lcll}
\hline $\mathrm{b}$ & $\mathrm{Q}$ & $\mathrm{v}$ & $\mathrm{F}$ \\
\hline 1.1 & 493.868 & 48.2375 & 46235.7 \\
1.2 & 1808.55 & 48.2375 & 51991.2 \\
1.3 & 2707.01 & 48.2375 & 44797.7 \\
1.4 & 3321.02 & 48.2375 & 34730.1 \\
1.5 & 3740.65 & 48.2375 & 25469.8 \\
1.6 & 4027.42 & 48.2375 & 18044.9 \\
1.7 & 4223.4 & 48.2375 & 12468.1 \\
1.8 & 4357.33 & 48.2375 & 8427.93 \\
\hline
\end{tabular}

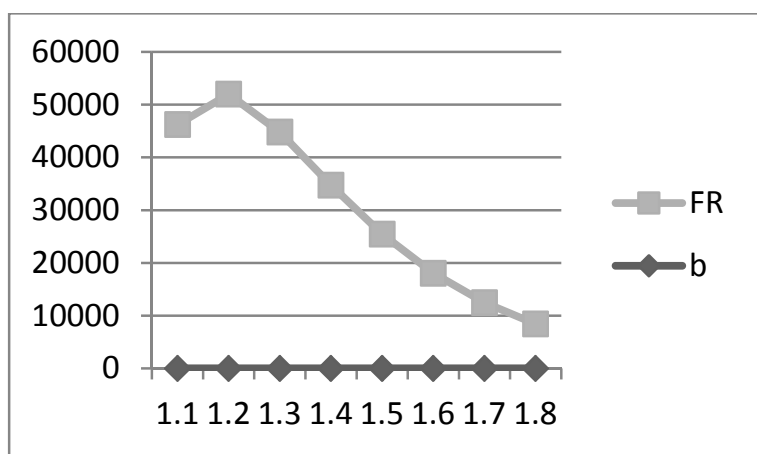

Variation in $F_{R}$ with the variation in $b$

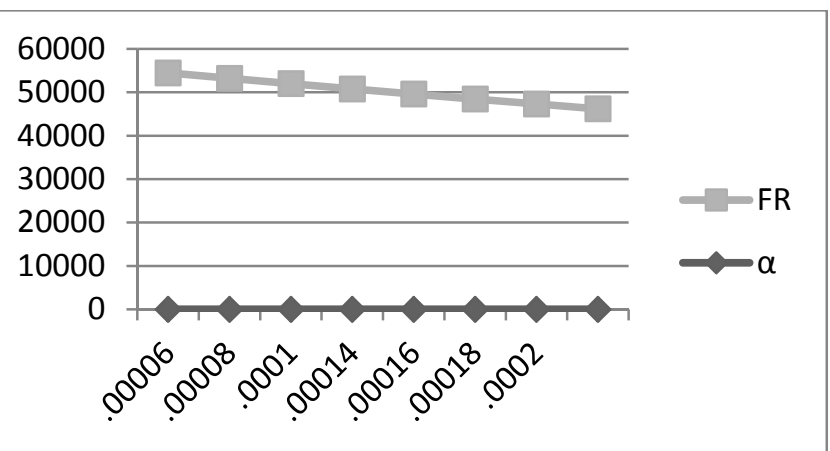

Variation in $F_{R}$ with the variation in $\alpha$

Table 10

Sensitivity analysis for the parameter $\alpha$

\begin{tabular}{lccc}
\hline$\alpha$ & $\mathbf{Q}$ & $\mathbf{v}$ & $\mathbf{F}_{\mathbf{R}}$ \\
\hline 0.00006 & 1901.41 & 48.2375 & 54471.8 \\
0.00008 & 1854.89 & 48.2375 & 53219.6 \\
0.0001 & 1808.55 & 48.2375 & 51991.2 \\
0.00012 & 1762.39 & 48.2375 & 50786.6 \\
0.00014 & 1716.41 & 48.2375 & 49605.5 \\
0.00016 & 1670.61 & 48.2375 & 48447.9 \\
0.00018 & 1624.99 & 48.2375 & 47313.7 \\
0.0002 & 1579.56 & 48.2375 & 46202.9 \\
\hline
\end{tabular}

\section{Table 11}

Sensitivity analysis for the parameter $\mathrm{y}(\mathrm{y}>0)$

\begin{tabular}{lccc}
\hline $\mathrm{y}$ & $\mathrm{Q}$ & $\mathrm{v}$ & $\mathrm{F}_{\mathrm{RD}}$ \\
\hline 5 & 1808.55 & 48.2375 & 51963.2 \\
10 & 1808.55 & 48.2375 & 51936.8 \\
15 & 1808.55 & 48.2375 & 51910.6 \\
20 & 1808.55 & 48.2375 & 51884.6 \\
25 & 1808.55 & 48.2375 & 51858.7 \\
30 & 1808.55 & 48.2375 & 51832.8 \\
35 & 1808.55 & 48.2375 & 51807 \\
40 & 1808.55 & 48.2375 & 51781.2 \\
45 & 1808.55 & 48.2375 & 51755.5 \\
\hline
\end{tabular}




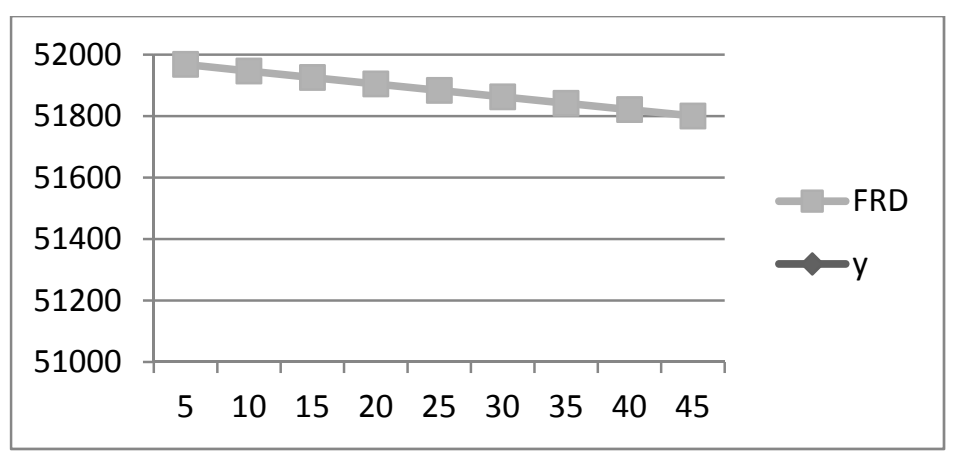

Variation in $\mathrm{F}_{\mathrm{RD}}$ with the variation in $\mathrm{y}$

\subsection{Discussion}

1. Table 2 shows the expiration date $(\mathrm{N})$ at $10,15,20,25,30$ and 35 and other variables unchanged. It is shown that as $\mathrm{N}$ increases, the replenishment cycle $\mathrm{nT}$ increases, and the retailer unit time profit $\left(\mathrm{F}_{\mathrm{R}}\right)$ decreases as well.

2. Table $3 \& 8$ shows the initial demand rate(a) in different cases, as the value of a increases, the value of unit time profit $\left(\mathrm{F}_{\mathrm{R}}\right)$ increases up to a fix point and after it this shows the reverse effect.

3. Table $4 \& 9$ lists the variation in $\mathrm{b}$ at $1.1,1.2,1.3,1.4,1.5,1.6,1.7$ and 1.8. Up to the value 1.2 unit time profit increases and after it the increase in $b$ results the decrease in $F_{R}$.

4. Table $5 \& 10$ lists the initial deterioration $\operatorname{rate}(\alpha)$ at different points and other variables unchanged. It shows that as $\alpha$ increases $\mathrm{Q}$ and $\mathrm{F}_{\mathrm{R}}$ decrease.

5. From table $6 \& 11$ we observe that as lead time(y) increases in different cases, the values of $\mathrm{Q}$ and $\mathrm{v}$ remain unchanged and unit time profit $\left(\mathrm{F}_{\mathrm{Rd}}\right)$ decreases respectively.

6. Table 7 shows the solution search results for the retailer when backlogs are not cleared due to logistic restrictions.

\section{Conclusion}

In this paper we have considered a supply chain model for two potential markets. In which demand is seasonal pattern and price dependent. Here a strategy for optional secondary market is used to reduce the cost of deterioration and warehouses. From our analysis, it is demonstrated that the retailer's profit is highly influenced by the supplier's lead time. The distributor has a single option of changing the market and/or the selling price. A solution procedure is provided for finding the optimal policy and optimal values of the decision variables. Numerical example shows that the model is effective. Our numerical investigations indicate that it will be beneficial for the distributor to shift to the alternate market at the earliest possible opportunity. For future research, we can extend this model for ramp type demand and time value of money.

Appendix A: Proof of theorem1:

$$
\begin{aligned}
& \frac{\partial F(v)}{\partial v}=\frac{p_{2} a}{N p_{2}{ }^{b}}(N-n+1)-\frac{\lambda p_{2} a}{p_{2}{ }^{b} T} v+\frac{a}{p_{2}{ }^{b}} v c-\frac{a}{p_{2}{ }^{b}}\left(\frac{v}{T}-1\right) r+\frac{a}{N p_{2}{ }^{b}}(N-n+1) d \\
& -\frac{s a}{p_{2}{ }^{b}}(T-v)-h a \frac{N-n+1}{N p_{2}{ }^{b}}\left(v+\alpha v^{\beta+1}-\frac{\alpha}{(\beta+1)} v^{\beta+1}\right) \\
& \frac{\partial^{2} F(v)}{\partial v^{2}}=-\frac{\lambda p_{2} a}{p_{2}{ }^{b} T}+\frac{a c}{T p_{2}{ }^{b}}-\frac{a}{p_{2}{ }^{b} T} r-\frac{h a(N-n+1)}{N p_{2}{ }^{b}}\left(1+\alpha \beta v^{\beta}\right)
\end{aligned}
$$


$\frac{\partial^{2} F(v)}{\partial v^{2}}=\frac{a}{p_{2}{ }^{b} T}\left(c-\lambda p_{2}\right)-\frac{a}{p_{2}{ }^{b} T} r-\frac{h a(N-n+1)}{N p_{2}{ }^{b}}\left(1+\alpha \beta v^{\beta}\right)$

Since $\mathrm{c}<\lambda p_{2}$ and $\mathrm{n}<\mathrm{N}, \Rightarrow \frac{\partial^{2} F(m, n, v)}{\partial v^{2}}<0$

It proves that $F(m, n, v)$ is concave in $\mathrm{v}$.

\section{Acknowledgement}

The authors wish to express gratitude the reviewers for their cooperative and valuable suggestions.

\section{References}

Banerjee, S., \& Sharma A. (2010). Inventory model for seasonal demand with option to change the market. Computers \& Industrial Engineering, 59, 807-818.

Bradely, J., \& Arntzen, B. (1999). The simultaneous planning of production, capacity and inventory in seasonal demand environments. Operations Research, 47, 6, 795-806.

Hsu, P.H., Wee, H.M., \& Teng, H.M. (2007). Optimal ordering decision for deteriorating items with expiration date and uncertain lead time. Computers \& Industrial Engineering, 52, 448-458.

Petruzzi, N. C., \& Monahan, G. (2003). Managing fashion goods inventories: Dynamic recourse for retailers with outlet stores. IIE Transaction, 35,1033-1047.

Pattnaik, M. (2013). Optimization in an instantaneous economic order quantity (EOQ) model incorporated with promotional effort cost, variable ordering cost and units lost due to deterioration, Uncertain Supply Chain Management, 1(4),57-66.

Saha, S., \& Basu, M. (2010),Integrated dynamic pricing for seasonal products with price and time dependent demand. Asia-Pacific Journal of Operational Research, 27(3), 393-410.

Saha, S., Das, S., \& Basu, M. (2010), Optimal pricing and production lot-sizing for seasonal products over a finite horizon. International Journal of Mathematics in Operational Research, 2(5), 540553.

Shukla, H.S., Shukla, V., \& Yadav, S.K. (2013). EOQ model for deteriorating items with exponential demand rate and shortages. Uncertain Supply Chain Management,1(2), 67-76.

Singh, S.R., \& Singh, N. (2009), A production inventory model with variable demand rate for deteriorating items under permissible delay in payments, International Transactions in Mathematical Sciences \& Computer, 2, 1, 73-82.

Singh, S.R., Kumar, T., \& Gupta, C.B. (2011). A soft computing based Inventory model with deterioration and price dependent demand. International Journal of Computer Applications, 36(4), 10-17.

Singh, S.R., \& Sharma S. (2014). Optimal trade-credit policy for perishable items deeming imperfect production and stock dependent demand. International Journal of Industrial Engineering Computations, 5(1), 151-168.

Singh, T.J., Singh, S.R., \& Dutt, R. (2009). An EOQ model for perishable items with power demand and partial backlogging. International Journal of Production Economics, 15(1), 65-72.

Singh, S.R., \& Singh, T.J. (2007). An EOQ inventory model with Weibull distribution deterioration, ramp type demand and partial backlogging. Indian Journal of Mathematics and Mathematical Sciences, 3(2), 127-137.

Singhal, S., \& Singh, S.R. (2013).Volume flexible multi items inventory system with imprecise environment. International Journal of Industrial Engineering Computations, 4(1), 457-468.

Tayal, S., Singh, S.R., Sharma, R., \& Chauhan, A. (2014).Two echelon supply chain model for deteriorating items with effective investment in preservation technology. International Journal of Mathematics in Operational Research, 6(1), 84-105. 
Tayal, S., Singh, S.R., \& Sharma, R. (2014). A multi item inventory models for deteriorating items with expiration date and allowable shortages. Indian Journal of Science and Technology, 7(4), 463-471.

Tripathi, R.P., \& Mishra, S.M. (2014). Inventory model with inventory-dependent demand for deteriorating items in a single warehouse system. Uncertain Supply Chain Management, 2(4), 209218.

Whitin, T. M. (1955). Inventory control and price theory. Management Science, 2, 61-68.

You, P. S. (2005). Optimal replenishment policy for product with seasonal pattern demand, Operations Research Letters, 33, 90-96. 